\title{
Die klimaresiliente Gesellschaft - Transformation und Systemänderungen
}

Jesko Hirschfeld, Gerrit Hansen, Dirk Messner

31.1 Kausalzusammenhang zwischen Klimaschutz und Anpassung, Anpassungsgrenzen und Transformation - 316

31.2 Globale Veränderungsprozesse und Transformation - 317

31.3 Chancen und Risiken der Anpassung in komplexen Systemen - 320

31.4 Kurz gesagt - 321

Literatur - 321 
Der Klimawandel stellt die Gesellschaft vor enorme Herausforderungen, die mehr erfordern werden als kleine Schritte der Anpassung in einzelnen Sektoren oder Regionen. Um langfristig „klimaresilient“ zu werden, wird eine weitreichende Transformation von Wirtschaft und Gesellschaft notwendig sein (Walker et al. 2004; Folke et al. 2010; IPCC 2014c). Diese Transformation wird sowohl aus der Perspektive des Klimaschutzes als auch aus der Perspektive der Anpassung an den Klimawandel notwendig werden und neben technologischen und wirtschaftlichen Anpassungen gesellschaftliche, kulturelle und politische Veränderungsprozesse erfordern (WBGU 2011).

In den nachfolgenden Unterkapiteln erörtern die Autoren die Zusammenhänge zwischen Klimaschutz und Anpassung an den Klimawandel, die Chancen, Risiken und Grenzen der Anpassung sowie den nationalen und globalen Transformationsbedarf. Es wird davor gewarnt, Klimaschutz und Anpassung an den Klimawandel als einfache Substitute zu betrachten und die Möglichkeiten von Anpassung zu überschätzen.

Neben den einzel- und volkswirtschaftlichen Auswirkungen des Klimawandels betrachten die Autoren auch die sozialen, politischen und ökologischen Auswirkungen in einem systemischen Zusammenhang und weisen zudem darauf hin, wie wichtig und schwierig räumliche und zeitliche Differenzierung sein kann.

\subsection{Kausalzusammenhang zwischen Klimaschutz und Anpassung, Anpassungsgrenzen und Transformation}

Klimaanpassung und Klimaschutz sind als komplementäre Maßnahmen zur Vermeidung negativer Klimawandelfolgen eng aneinander gekoppelt. Klimaschutzmaßnahmen sind, zeitverzögert, entscheidend für das Ausmaß des Klimawandels und damit auch für die notwendige Anpassung, während Kosten und Potenziale von Anpassung bestimmend für Klimaschutzanstrengungen sein können. Daneben konkurrieren sie um ähnliche Ressourcen und sind durch Synergien und Trade-offs verbunden (Klein et al. 2007; Moser 2012). Oft werden beide Maßnahmengruppen als Substitute behandelt, was jedoch die Gefahr birgt, wichtige Interaktionen sowie mögliche Grenzen der Anpassung zu ignorieren. Um Kosten und Risiken des Klimawandels zu reduzieren, ist ein aufeinander abgestimmter Mix aus ehrgeizigen Klimaschutzzielen und nachhaltigen Anpassungsmaßnahmen wichtig (IPCC 2014c).

Verschiedene Emissionspfade führen zu unterschiedlichen Klimafolgen und Unsicherheitsniveaus hinsichtlich der Wirksamkeit von Anpassungsmaßnahmen (IPCC 2013; Kap. 2). Die langfristige klimatische Entwicklung wird in hohem Maße davon abhängen, welche Klimaschutzanstrengungen unternommen werden. Die Anpassung an die bereits im Klimasystem eingeschriebene Erwärmung von bis zu ca. $2{ }^{\circ} \mathrm{C}$ gegenüber vorindustriellen Temperaturen muss hingegen in jedem Falle geleistet werden. Während bei den gemäßigten Emissionsszenarien eine Restabilisierung des Klimas auf höherem Niveau gegen Ende dieses Jahrhunderts projiziert wird, sind bei Szenarien mit höheren Emissionen auch nach 2100 noch langfristige und fundamentale Veränderungen zu erwarten (IPCC 2013). Entsprechend sind die mit geringeren Klimaschutzanstrengungen verbundenen Szenarien höherer Emissionen nicht nur mit massiveren Auswirkungen, sondern auch mit größeren Unsicherheiten bezüglich der langfristig notwendigen Anpassungsleistungen behaftet.

Kurz- und mittelfristige Anpassungsmaßnahmen zielen oft auf die Verwirklichung sogenannter low- und no-regret-Optionen, die eine Verbesserung der Resilienz bezüglich verschiedener zukünftiger Klimaszenarien zum Ziel haben, oft unter gleichzeitiger Erfüllung anderer relevanter Politikziele ( Kap. 30; Hallegatte 2009). Insbesondere für langfristige Investitionsentscheidungen mit langen Vorlaufzeiten - z. B. Küsten- und Hochwasserschutz, Forstwirtschaft, Energieerzeugung sowie Siedlungs- und Infrastrukturplanung - kann die klimawandelbedingte Planungsunsicherheit jedoch zu einem erhöhten Risiko von Fehlinvestitionen und damit mittelbar zu steigenden Kosten führen. Robuste Anpassung in diesem Bereich bedarf daher neuer, schrittweiser Planungsverfahren und muss ein breites Band von möglichen „Klimazukünften“ berücksichtigen (Hallegatte 2009; Dessai und Hulme 2007; Wilby und Dessai 2010). Klimaschutzanstrengungen sind ein entscheidender Faktor für die Breite dieses Bandes und wirken damit auf die Kosten und Realisierbarkeit von Anpassungsmaßnahmen (Hallegatte et al. 2012).

Global sind die zu erwartenden Kosten von Anpassung bisher unzureichend quantifiziert. Existierende Abschätzungen fokussieren auf Entwicklungsländer (World Bank 2010) und einzelne Sektoren wie z. B. Küstenschutz, Wasser- und Energieversorgung sowie Landwirtschaft (Fankhauser 2010). Globale ökonomische Schadenskosten sind unvollständig und aufgrund von zahlreichen Annahmen und hoher Aggregation wenig aussagekräftig beschrieben (IPCC 2014c). Insbesondere für Klimaszenarien jenseits von $3{ }^{\circ} \mathrm{C}$ Erwärmung existieren zudem kaum aktuelle Studien. Die in der Literatur dagegen ausführlich dokumentierten Klimaschutzkosten für verschiedene Emissionsszenarien beruhen zum Großteil auf integrated-assessment-Modellen ( Kap. 25). Diese bilden die Veränderungen im Energiesystem und anderen Sektoren sowie die damit einhergehenden Kosten und Veränderungen in der Weltwirtschaft ab. Sie berücksichtigen jedoch meist weder verbleibende Schadenskosten noch Kosten der Anpassung oder Rückkopplungen im Klimasystem explizit (Patt et al. 2010).

Diese Problematik der mangelnden Integration von Anpassung, Schadenskosten und Klimaschutz bestand schon im Vierten Sachstandsbericht (Parry 2009), und es gibt nach wie vor nur wenige globale Studien zu deren integrierter Kostenabschätzung (Bosello et al. 2010; de Bruin et al. 2009; Kap. 25). Trotz methodischer Fortschritte - etwa die bessere Repräsentation von Anpassung in integrated-assessment-Modellen durch das Vergleichen entsprechender sozioökonomischer Szenarien (Kriegler et al. 2012; van Vuuren et al. 2011) - ist die integrierte Modellierung von Klimaschutz-, Schadens- und Anpassungskosten derzeit noch in ihren Anfängen (Fisher-Vanden et al. 2013; Patt et al. 2010; Hirschfeld et al. 2015). Kritisch wird dabei die zentrale Rolle der in gewissem Maße beliebig gewählten Diskontrate zur Bestimmung gesellschaftlich optimaler Transformationspfade diskutiert (Guo et al. 2006; Weitzman 2013), da hohe Diskontraten (>3\%) zu einer weitgehenden Vernachlässigung von Klimakosten führen, die mehr als wenige Jahrzehnte in der 
Zukunft liegen. Darüber hinaus hat die Schwierigkeit, das Risiko von katastrophalen Klimawandelfolgen adäquat zu berücksichtigen (Weitzman 2009), zu grundsätzlicher Kritik an der Eignung solcher Modelle als Grundlage für politische Entscheidungen geführt (Fisher-Vanden et al. 2013; Stern 2013).

Die gesellschaftlich optimale Mischung von Anpassung und Klimaschutz lässt sich demnach nach wie vor nicht aus Ergebnissen ökonomischer Modelle herleiten. Der Umgang mit „Unquantifizierbarkeiten“ kann am ehesten mit einem gekoppelten Ansatz der Risikoanalyse erfolgen, wobei Methoden wie Multikriterienanalysen zur besseren Erfassung der Dimensionen von Risiko und Unsicherheit beitragen können (Vetter und Schauser 2013; van Ierland et al. 2013; Kap. 32). Hierbei können neben grundsätzlichen ethischen Überlegungen auch Risiken von Klimaschutzmaßnahmen einfließen, z. B. die Herausforderungen großskaliger Bioenergieverwendung (Chum et al. 2011), negative ökonomische Folgen für arme Länder (Jakob und Steckel 2014) oder die Risiken von „Geo-Engineering“ (IPCC 2012). Zentrale Elemente einer solchen Risikoabschätzung sind das Risikominderungspotenzial und die Grenzen der Anpassung.

Harte, d.h. unveränderliche Grenzen stellen insbesondere die sogenannten Kipppunkte dar (Lenton et al. 2008; Levermann et al. 2012): großskalige, sprunghafte Zustandsänderungen von wichtigen Elementen des Erdsystems. Neben den Kipppunkten des physikalischen Klimasystems ist hier die erhöhte Gefahr sogenannter regime shifts, also dauerhafter Umwälzungen in den komplexen Mustern der global bedeutenden Ökosysteme relevant. So sind in der Arktis bereits Hinweise auf ein Überschreiten von Systemgrenzen und damit die Annäherung an Kipppunkte zu erkennen (Duarte et al. 2012; Lenton 2012; Post et al. 2009; Wassmann und Lenton 2012). Die Schädigung von tropischen Korallenriffen ist ebenfalls bereits nachgewiesen (Cramer et al. 2014), und die Gefahr des kompletten Verlustes dieses komplexen Ökosystems besteht selbst bei geringer weiterer Erwärmung (Frieler et al. 2012; Hoegh-Guldberg 2011), verbunden mit erheblichen Risiken für die Nahrungsversorgung, nicht nur von Küstenbewohnern. Auch die negativen Folgen der zunehmenden Ozeanversauerung stellen aufgrund der mangelnden Anpassungsmöglichkeiten eine harte Grenze dar (Pörtner et al. 2014). Sogenannte weiche Grenzen der Anpassung bezeichnen Bereiche, in denen die Klimawandelfolgen zwar theoretisch als technisch beherrschbar eingeschätzt werden, es aber Ziel- oder Wertkonflikte gibt, welche die Umsetzung entsprechender Maßnahmen behindern oder diese auf institutioneller, politischer oder gesellschaftlicher Ebene nicht durchführbar oder durchsetzungsfähig erscheinen lassen (Preston et al. 2013). Ein Beispiel sind Maßnahmen des Hochwasserschutzes, die nicht nur mit den Zielen des Küsten- und Naturschutzes kollidieren können, sondern auch mit den Interessen von Anwohnern und der Tourismusindustrie (Moser et al. 2012). Die Forschung hierzu steht, ähnlich wie diejenige zur Interaktion verschiedener Klimawandelfolgen (Warren 2011), noch am Anfang - insbesondere bezüglich der sozialen Grenzen von Anpassung und der Wechselwirkung zwischen sozialen und natürlichen Systemen (Adger et al. 2009; Preston et al. 2013).

Der Weltklimarat nimmt im Fünften Sachstandsbericht eine umfassende Bewertung von regionalen und globalen Schlüssel- risiken unter verschiedenen Erwärmungs- und Anpassungsszenarien vor (IPCC 2014c). Das theoretische Potenzial von Anpassung zur Risikominderung für Europa wird dabei - isoliert betrachtet, also unabhängig von Kosten und politischen Prioritäten - insgesamt auch in einer Vier-Grad-Welt als relativ hoch eingeschätzt (- Abb. 31.1). Im Vergleich zu einer Zwei-Grad-Welt ist die Erschließung dieses theoretisch vorhandenen Potenzials jedoch mit entsprechend höherem Aufwand und höherer Unsicherheit verbunden. In anderen Kontinenten und für bestimmte Sektoren, auch für Subregionen in Europa, sind die verbleibenden Restrisiken selbst bei optimaler Anpassung in einer VierGrad-Welt dagegen teilweise hoch oder sehr hoch und damit in der Nähe harter Grenzen (• Abb. 31.1).

Auch wenn die Gesamtkosten von Klimaschutzanstrengungen, die noch immer zum Erreichen des Zwei-Grad-Ziels führen könnten, relativ gering ausfallen, wenn sie frühzeitig, unter Einbindung sämtlicher technologischen Möglichkeiten und global erfolgen (Luderer et al. 2013; IPCC 2014a), sind die Anforderungen, um zu einer Zwei-Grad-Welt zu kommen, doch erheblich ( Abschn. 31.2), zumal das dazu notwendige globale Einvernehmen hinsichtlich der Selbstverpflichtung zur Einhaltung ehrgeiziger Ziele nicht ausreicht - die Grenzen müssen ja auch eingehalten werden. So kann zumindest temporär ein Überschreiten der Zwei-Grad-Grenze nicht ausgeschlossen werden, ein sogenanntes overshoot-Szenario eintreten (Parry et al. 2009). Zudem droht beim Fortschreiben der derzeitigen Emissionstrends eine Erwärmung von mehr als $4^{\circ} \mathrm{C}$ bis Ende des Jahrhunderts (IPCC 2013). Dort wo infolge solch starker Erwärmung Maßnahmen zur inkrementellen Anpassung nicht mehr ausreichen, würden transformative Anpassungsstrategien notwendig werden, die einen weitreichenden Wandel wirtschaftlicher, sozialer und politischer Systeme beinhalten (Smith et al. 2011; Kap. 33).

Für eine integrierte Betrachtung der Risiken und Kosten von Klimawandel und Klimaschutz besteht nach wie vor erheblicher Forschungsbedarf, insbesondere bezüglich der Operationalisierung und Quantifizierung von Anpassung und Schadenskosten. Nach derzeitigem Stand der Forschung ist allerdings klar, dass ambitionierte Klimaschutzanstrengungen unverzichtbar sind, um schwerwiegende und weitreichende globale Klimawandelfolgen abzuwenden (IPCC 2014b).

\subsection{Globale Veränderungsprozesse und Transformation}

Alleinige, radikale Reduzierungen der Treibhausgasemissionen der OECD-Länder reichen nicht mehr aus, um die Zwei-GradLeitplanke einzuhalten. Zwischen 1990 und 2010 sind deren jährliche Treibhausgasemissionen von gut 10 auf knapp 14 Gigatonnen gestiegen und stagnieren seitdem in etwa auf diesem Niveau. Die Emissionen der Nicht-OECD-Länder haben sich im gleichen Zeitraum von 10 auf 20 Gigatonnen erhöht, insbesondere infolge des hohen Wachstums in den Schwellenländern. Setzen sich die derzeitigen Trends fort, so dürften sich die OECD-Emissionen pro Jahr zwischen 2010 und 2040 auf einem Niveau von etwa 15 Gigatonnen einpendeln, während die Emissionen pro Jahr der 


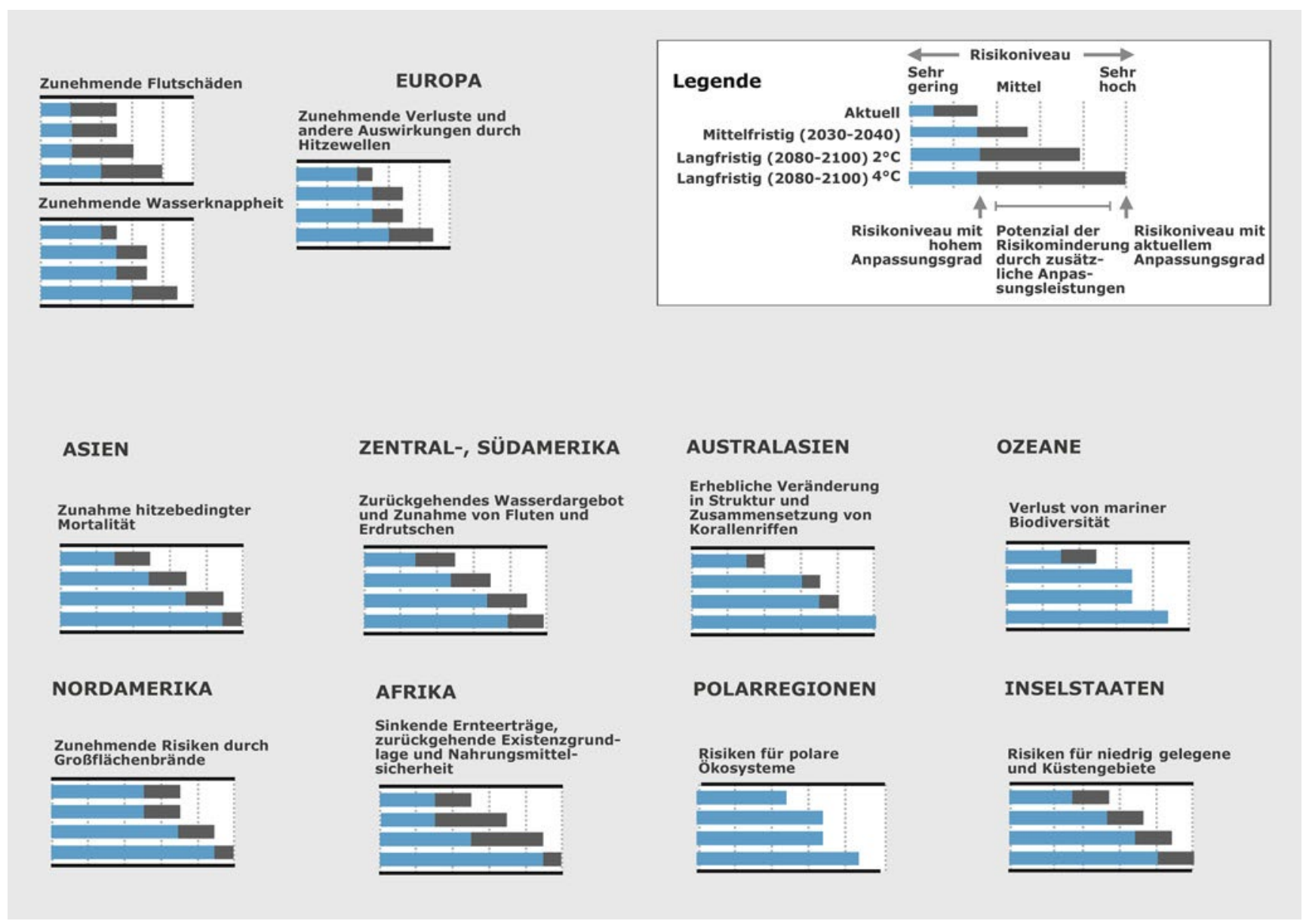

- Abb. 31.1 Schlüsselrisiken für Europa auf verschiedenen Zeitskalen. Beispiele für Schlüsselrisiken mit hohem oder sehr hohem Risiko und geringer Anpassungskapazität bei starker Erwärmung in Weltregionen außerhalb Europas (nach IPCC 2014c). Balken zeigen das Risiko unter Annahme aktueller (gesamter Balken) oder optimaler Anpassung (blauer Balken), gegenwärtig, mittelfristig (2030-2040) sowie langfristig (2080-2100) für eine potenzielle Zwei-Grad- und Vier-Grad-Welt. (Nach Kovats et al. 2014, Tab. 23-5)

Nicht-OECD-Länder in diesem Zeitraum von 20 auf gut 30 Gigatonnen ansteigen würden (IEA 2013). Weil sich die Dynamiken in der Weltwirtschaft in den vergangenen zwei Jahrzehnten signifikant in Richtung der Nicht-OECD-Länder verschoben haben (OECD 2010; UNDP 2013; Kaplinsky und Messner 2008; Spence 2011), ist wirksamer Klimaschutz nur noch möglich, wenn die grundlegenden Wachstumsmuster aller Länder auf einen klimaverträglichen Pfad gebracht werden.

Globaler Klimaschutz ist daher zu einem Synonym für den Aufbau einer global low carbon economy (Edenhofer et al. 2009; Leggewie und Messner 2012; World Bank 2012) geworden. Neben der Frage, wie radikale Dekarbonisierung in OECD-Ländern gelingen kann, besteht die zweite Herausforderung darin, wie zunehmender Wohlstand in den Nicht-OECD-Ländern von Treibhausgasemissionen entkoppelt werden kann (Kharas 2010). Diese sozioökonomischen Dynamiken globalen Wandels werden auch in der internationalen Politik sichtbar, etwa in Diskussionen darüber, wie das „Recht auf Entwicklung“ mit globalem Klimaschutz verbunden werden kann (Pan 2009; WBGU 2009). Globale Gerechtigkeits- und Verteilungsfragen bilden vor diesem Hintergrund eine zentrale Arena der weltweiten Klimapolitik und der Versuche, einen Übergang zu einer klimaverträglichen Ökonomie einzuleiten (Gesang 2011).
Der Übergang zu einer klimaverträglichen Wirtschaft wird in der Literatur zunehmend aus der Perspektive von Transitionsbzw. Transformationsprozessen diskutiert, um zu verdeutlichen, dass der Umbruch zu einer low carbon economy über klassische Muster des Strukturwandels (Transition) in einzelnen Marktwirtschaften hinausgeht und umfassende Prozesse des Wandels (Transformation) impliziert (Rotmans et al. 2001; Martens und Rotmans 2002; Grin et al. 2010; World Bank 2012; Brand et al. 2013). Der Wissenschaftliche Beirat der Bundesregierung Globale Umweltveränderungen (WGBU) hat vorgeschlagen, den Übergang zu einer klimaverträglichen und insgesamt nachhaltigen Weltwirtschaft als „große Transformation“ zu beschreiben (WBGU 2011) und verweist auf fünf Argumentationsstränge, die aus der Perspektive des Wissenschaftlergremiums gute Gründe für diese Benennung liefern:

- Der Übergang zur Klimaverträglichkeit kann nur gelingen, wenn die globalen Wachstumsmuster in Richtung Dekarbonisierung verändert werden - wenn also ein neuer Pfad globaler Entwicklung eingeschlagen wird. Ob diese Weichenstellung gelingt, hängt einerseits davon ab, ob in den Industrieländern der Übergang zur Klimaverträglichkeit eingeleitet wird. Andererseits wird es von großer Bedeutung sein, ob die dynamisch wachsenden Schwellen- 
länder bereit und in der Lage sind, Dekarbonisierung in das Zentrum ihrer Entwicklungsanstrengungen zu rücken (IPCC 2014c, Working Group III). Eine solche Veränderung von Wachstumsmustern setzt eine grundlegende Transformation institutioneller Rahmenbedingungen voraus, um Anreize für klimaverträgliche Investitionen zu schaffen (World Bank 2012; Edenhofer und Stern 2009; Schmitz et al. 2013; Global Commission on the Economy and Climate 2014).

- Die Entwicklung einer klimaverträglichen Weltwirtschaft impliziert einen weitgehenden Umbau der zentralen Infrastrukturen u. a. hin zu ressourcensparenden und klimarobusten Systemen, auf denen menschliche Gesellschaften basieren: in den weltweiten Energiesystemen, die für etwa $75 \%$ der globalen Treibhausgasemissionen verantwortlich sind, in der Landnutzung (Waldnutzung, Landwirtschaft), auf die etwa $25 \%$ der Emissionen entfallen, und in urbanen Räumen, weil ein großer Teil der Emissionen auf die Bedürfnisfelder Wohnen (Gebäude) und Mobilität in Städten zurückzuführen ist (Nakicenovic et al. 2000; WBGU 2011; Urban und Nordensvard 2013; IPCC 2014a). Die Urbanisierung ist von besonderer Bedeutung, weil die Zahl der Menschen, die in urbanen Räumen lebt, von derzeit $3 \mathrm{Mrd}$. auf 6 Mrd. Menschen im Jahr 2050 ansteigen wird (UN Habitat 2011). Bei Gebäuden und Mobilitätssystemen handelt es sich um besonders pfadabhängige Infrastrukturen, welche die Emissionspfade für viele Jahrzehnte prägen werden (IEA 2010; EWI et al. 2010). Ob also der Urbanisierungsschub, der sich insbesondere auf Nicht-OECD-Länder und hier vor allem auf Asien konzentriert, low-carbon-Mustern oder den etablierten treibhausgasintensiven Dynamiken der Stadtentwicklung folgt, ist aus der Perspektive des Klimaschutzes von großer Bedeutung (WBGU 2011).

- Dekarbonisierungsstrategien müssen auf technologischen Innovationen basieren. Die Literatur zum rebound-Effekt (Jackson 2009; Nordhaus 2013) verdeutlicht allerdings auch, dass eine absolute Abkopplung der Wohlstandsentwicklung von Emissionen nur gelingen kann, wenn sich zugleich soziale Innovationen durchsetzen: veränderte Lebensstile und Konsummuster, neue Wohlfahrtskonzepte sowie Normen und Wertesysteme, die den Erhalt der globalen Gemeinschaftsgüter zu einem kategorischen Imperativ machen (Skidelsky und Skidelsky 2012; World Bank 2012; Messner 2015).

- Die Transformation muss in einem sehr engen Zeitfenster stattfinden, wenn das Zwei-Grad-Ziel noch eingehalten werden soll (Allen et al. 2009; Meinshausen et al. 2009; WBGU 2009). Bis etwa 2070 müssten die Treibhausgasemissionen, die aus der Verbrennung fossiler Energieträger entstehen, weltweit auf null reduziert werden (WBGU 2014). So stellt sich die Frage, wie und ob Dynamiken der Transformation beschleunigt werden können (Grin et al. 2010). Zugleich bewirken Treibhausgasemissionen in der Gegenwart langfristige Dynamiken im Erdsystem, bis hin zum Risiko des Erreichens von Kipppunkten (Lenton et al. 2008). Die für menschliche Gesellschaften relevanten Zeitregimes verändern sich daher, ähnlich wie während der industriellen Revolution (Osterhammel 2009; Sieferle et al. 2006; Leggewie und Messner 2012).

- Wenn Paul Crutzen und andere (Crutzen 2000; Williams et al. 2011) mit ihrem Argument recht behalten, dass die Menschheit zu einer zentralen Veränderungskraft im Erdsystem geworden ist, impliziert der Übergang zu einer nachhaltigen Wirtschafts- und Gesellschaftsordnung, dass die Menschen Institutionen sowie Normen und Wertesysteme „erfinden“ müssen, um das Erdsystem im Anthropozän - nach Paul Crutzen das Zeitalter der Menschen - dauerhaft zu stabilisieren und damit die Existenzgrundlagen vieler künftiger Generationen zu erhalten. Diese Herausforderungen eines „Erdsystemmanagements“ (Schellnhuber 1999; Biermann 2007, 2008) gehen über die existierenden Weltbilder internationaler Politik deutlich hinaus.

Der Verweis auf Klimaschutz und Anpassung an den Klimawandel im Kontext der Dynamiken globaler Entwicklung sowie die Diskussion über das Klimasystem als Gemeinschaftsgut (global common) (Ostrom 2010) führen zu der Frage, wie globale Kooperation gestaltet werden kann, um die Transformation zur Klimaverträglichkeit zu ermöglichen (Keohane und Victor 2010; Oberthuer und Gering 2005; Ostrom 2009; WBGU 2006; Messner und Weinlich 2016). In der Literatur wird auf vier zentrale Mechanismen verwiesen, welche die Klimaverhandlungen schwierig und langwierig mach(t)en:

1. auf das aus der Konzeption der tragedy of the commons (Hardin 1968), die auf die Gefahr der Übernutzung frei verfügbarer und begrenzter Ressourcen verweist, und der Theorie kollektiven Handelns (Olson 1965) bekannte „Trittbrettfahrerproblem“ (Nordhaus 2013). Es bedeutet, dass das Zustandekommen von Kooperationsallianzen (z. B. zum Schutz des Klimasystems) erschwert wird, wenn Akteure, die sich nicht an diesen kooperativen Lösungen beteiligen, nicht an der weiteren Übernutzung bzw. Überlastung des Gemeinschaftsgutes gehindert werden können;

2. auf Verteilungskonflikte zwischen Industrie-, Schwellen- und Entwicklungsländern über die Kosten, die durch Treibhausgasreduzierungen entstehen, sowie über Verantwortlichkeiten zur Treibhausgasminderung, die sich für jeweilige Länder(gruppen) aus historischen, gegenwärtigen und zukünftig zu erwartenden Emissionen ergeben (WBGU 2009; Ott et al. 2008, Depledge 2005; Pan 2009);

3. auf die Sorge von Entscheidungsträgern, dass radikale Treibhausgasreduzierungen die Wettbewerbsfähigkeit ihrer Ökonomien schädigen, Beschäftigungseinbußen zur Folge haben oder - so der Diskurs in Schwellen- und Entwicklungsländern - Prozesse nachholender Entwicklung blockieren könnten (Leggewie und Messner 2012; World Bank 2012; OECD 2010; Sinn 2008);

4. auf die spezifische Zeitstruktur des Klimaproblems, die darin besteht, dass schwerwiegende Folgen des Klimawandels erst in einigen Jahrzehnten zu erwarten sind, politische Systeme und Entscheidungsträger jedoch primär auf aktuellen Problemdruck reagieren (Giddens 2009; Newton-Smith 1980; Zimmerman 2005; WBGU 2014). 


\begin{tabular}{|c|c|c|c|c|c|}
\hline \multirow[b]{3}{*}{$\begin{array}{l}\text { Effekte der } \\
\text { Anpassung }\end{array}$} & \multicolumn{5}{|c|}{ Systemdimensionen } \\
\hline & \multicolumn{2}{|c|}{ wirtschaftlich } & \multirow[t]{2}{*}{ sozial } & \multirow{2}{*}{$\begin{array}{c}\text { politisch/ } \\
\text { institutionell }\end{array}$} & \multirow[t]{2}{*}{ ökologisch } \\
\hline & $\begin{array}{c}\text { einzel- } \\
\text { wirtschaftlich }\end{array}$ & $\begin{array}{c}\text { gesamt- } \\
\text { wirtschaftlich }\end{array}$ & & & \\
\hline Chancen & $\begin{array}{l}\text { Gewinne, } \\
\text { vermiedene } \\
\text { Schäden }\end{array}$ & $\begin{array}{l}\text { Wohlfahrts- } \\
\text { gewinne }\end{array}$ & ausgleichend & $\begin{array}{l}\text { konflikt- } \\
\text { reduzierend }\end{array}$ & $\begin{array}{c}\text { positive } \\
\text { ökosystemare } \\
\text { Wirkungen }\end{array}$ \\
\hline Risiken & $\begin{array}{l}\text { Schäden, } \\
\text { Verluste }\end{array}$ & $\begin{array}{l}\text { Wohlfahrts- } \\
\text { verluste }\end{array}$ & polarisierend & $\begin{array}{c}\text { konflikt- } \\
\text { verschärfend }\end{array}$ & $\begin{array}{c}\text { negative } \\
\text { ökosystemare } \\
\text { Wirkungen }\end{array}$ \\
\hline \multirow{2}{*}{$\begin{array}{l}\text { Skalen- } \\
\text { ebenen }\end{array}$} & \multicolumn{3}{|c|}{$\longleftarrow$ räumlich } & 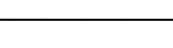 & $\rightarrow$ \\
\hline & \multicolumn{2}{|c|}{$\longleftarrow$} & zeitlich & & $\rightarrow$ \\
\hline
\end{tabular}

- Abb. 31.2 Chancen und Risiken der Anpassung auf verschiedenen Dimensionen komplexer Systeme (Eigene Darstellung)
Diese Kooperationshemmnisse sind Gründe dafür, dass die internationale Staatengemeinschaft 21 Verhandlungsrunden benötigte, um im Dezember 2015 in Paris einen alle Staaten in Verpflichtungen einbindenden Weltklimavertrag abzuschließen, obwohl die naturwissenschaftlichen Grundlagen des Klimawandels, seiner Ursachen, Treiber und Wirkungen seit geraumer Zeit gut verstanden und sogar von der überwiegenden Zahl der Staaten akzeptiert waren. Das Pariser Klimaabkommen sieht erstmals in der Geschichte der Klimadiplomatie eine Dekarbonisierung der Weltwirtschaft in der zweiten Hälfte des 21. Jahrhunderts vor. Der Klimavertrag stellt einen Versuch dar, die Handlungsblockaden, die aus den vier Kooperationshemmnissen resultieren, durch Kompromisse, Selbstverpflichtungen der Staaten, Ausgleichszahlungen, Technologietransfer und Monitoringsysteme für die Umsetzung der Vereinbarungen zu überwinden. Auf Sanktionsmechanismen für Kooperationsverweigerer und Staaten, die ihren Verpflichtungen nicht nachkommen, haben sich die Staaten nicht einigen können. Ob diese historische Neuorientierung in Richtung Dekarbonisierung in den kommenden Jahren auf nationaler und internationaler Ebene auch tatsächlich durchgesetzt wird, bleibt abzuwarten.

\subsection{Chancen und Risiken der Anpassung in komplexen Systemen}

Chancen und Risiken der Anpassung an den Klimawandel sind sowohl auf globaler Ebene als auch im nationalen Maßstab bislang unzureichend quantifiziert und werden auch in Zukunft nur in Grenzen quantifizierbar sein (Watkiss 2009; JPI Climate 2011; Defra et al. 2012; Hirschfeld et al. 2015; Schröder und Hirschfeld 2015). Das stellt nationale, regionale und lokale Entscheidungsträger vor teilweise erhebliche Probleme bei der Formulierung angemessener Anpassungspolitiken. Die Schwierigkeiten bei der Abbildung der Kosten und Nutzen von Anpassungsmaßnahmen ergeben sich zum einen aus den klima- und ökosystemaren Unsicherheiten und Ungewissheiten, mit denen Klimaszenarien nach wie vor behaftet sind und voraussichtlich auch dauerhaft sein werden. Zum anderen folgen sie aus der Komplexität der angesprochenen wirtschaftlichen, sozialen und politischen Systeme, die durch den Klimawandel zu reaktivem und proaktivem Handeln herausgefordert sind (WBGU 2011; Kap. 28).

Nach den bisher vorliegenden Analysen zu Kosten und Nutzen der Anpassung an den Klimawandel in Deutschland zeichnen sich die vordringlichsten Anpassungsbedarfe und größten erreichbaren Anpassungsnutzen in den Bereichen Hitze, Hochwasser und Stürme ab (Hübler und Klepper 2007; Robine et al. 2008; Hinkel et al. 2010; Tröltzsch et al. 2011; GDV 2013; Lehr und Nieters 2015; IPCC 2014b).

- Abb. 31.2 gibt einen Überblick zu den im Folgenden diskutierten Dimensionen komplexer Systeme (wirtschaftliche, soziale, politische, ökologische), die durch Maßnahmen zur Anpassung an den Klimawandel in positiver, neutraler oder negativer Weise beeinflusst werden können. Zur Abwägung zwischen Chancen und Risiken der Anpassung lassen sich die bereits erwähnten Kosten-Nutzen- und Multikriterienanalysen heranziehen. Letztere stellen die Effekte von Anpassungsmaßnahmen in der Vielfalt ihrer Dimensionen dar, ohne sie auf eine einheitliche Dimension von Geldwerten umzurechnen und damit unmittelbar vergleichbar zu machen.

Zusätzlich und über alle Systemdimensionen hinweg sind die räumlichen und zeitlichen Skalenebenen zu beachten. Es ist in vielen Fällen von hoher Relevanz für die Entscheidung über die Vorteilhaftigkeit einer Anpassungsmaßnahme, ob die Wirkungen der Maßnahme vor einem kleinräumig-lokalen Betrachtungshintergrund bewertet werden oder auf einer überregionalen, nationalen oder sogar globalen Skalenebene. Ebenso ist es häufig entscheidend, ob Wirkungen kurz-, mittel- oder langfristig betrachtet und in die Entscheidungsprozesse einbezogen werden.

Zur Entscheidungsfindung müssen die Systemdimensionen untereinander gewichtet werden. Außerdem sind für die einzelnen Dimensionen kritische Untergrenzen zu beachten, bei deren Unterschreitung die Stabilität der jeweiligen Systeme gefährdet 
wird (etwa einzelbetriebliche Rentabilität, sozialer Friede, Resilienz des betroffenen Ökosystems). Sowohl die Gewichtung als auch die Bezugnahme auf bestimmte räumliche und zeitliche Skalenebenen (lokal oder global, kurz- oder langfristig) können nur auf Grundlage von Werturteilen vorgenommen werden und sind damit im politischen Prozess zu treffende Entscheidungen. Anpassungsmaßnahmen an den Klimawandel sind also nicht allein aus individueller oder betriebswirtschaftlicher Perspektive zu betrachten, wenn unerwünschte Nebeneffekte oder sogar negative Gesamteffekte vermieden werden sollen (Hirschfeld und von Möllendorff 2015). Gleichzeitig stellen nur wenige Anpassungsmaßnahmen win-win-win-Lösungen für alle Akteursgruppen in allen Systemdimensionen und auf allen Skalenebenen dar. Häufig müssen in mindestens einer der Systemdimensionen Abstriche hingenommen werden, um die in einer anderen Dimension oder auf einer anderen Skalenebene gesetzten Ziele zu erreichen.

Bei der Gestaltung von Anpassungspolitiken sollten potenzielle Anpassungsmaßnahmen also im Hinblick auf ihre komplexen Auswirkungen in den verschiedenen Systemdimensionen analysiert und ihre Ansatzpunkte auf den verschiedenen räumlichen und zeitlichen Skalenebenen berücksichtigt werden. Entsprechend der Vielzahl der angesprochenen Systemdimensionen sind dabei die Zusammenarbeit zwischen verschiedenen sozialund naturwissenschaftlichen Forschungsdisziplinen sowie die Einbeziehung der jeweils betroffenen und handlungsrelevanten Akteursgruppen notwendig.

Auf einzelwirtschaftlicher Ebene begrenzen Budgetrestriktionen und teilweise abträgliche Anreizsituationen die Handlungsmöglichkeiten von Unternehmen und Haushalten. In vielen Fällen fehlt bislang auch das Wissen über geeignete Anpassungsoptionen. Hier können durch Informationsbereitstellung sowie geeignete institutionelle Rahmensetzungen Anreizmuster verändert und Möglichkeiten zu autonomen Anpassungsanstrengungen eröffnet werden ( Kap. 33). Staatliche Institutionen haben hierzu in den letzten Jahren eine Vielzahl von Aktivitäten zur Anpassung an den Klimawandel gestartet ( Kap. 32). Damit könnten bei Haushalten und Unternehmen Win-win-Potenziale gezielt erzeugt und genutzt werden.

Für Wirtschaftsverbände, Nichtregierungsorganisationen und Vereine gilt es, sich und seine Mitglieder über Klimafolgen und Anpassungsoptionen zu informieren, diese zu diskutieren und ihre gemeinsame oder auch individuelle Umsetzung beratend zu begleiten ( Kap. 32).

Die Forschung schließlich kann Praxisfragen, Wissensbedarfe und vorhandenes Systemwissen der in diesem Kapitel genannten Akteure, Beteiligten und Betroffenen aufnehmen und in einem inter- und transdisziplinären Forschungsprozess (Jahn 2008) Wissen über die potenziellen Folgen des Klimawandels und die Chancen und Risiken von Klimaanpassungsmaßnahmen auf den verschiedenen Ebenen komplexer wirtschaftlicher, sozialer, politischer und ökologischer Systeme erarbeiten. Auf dieser Grundlage können wissenschaftliches Wissen mit gesellschaftlichen Visionen und Wertvorstellungen zusammengeführt, Klimaserviceprodukte entwickelt und wissensbasierte Transformationen (WBGU 2011) in Richtung einer klimaresilienten Gesellschaft ausgehandelt und angestoßen werden.

\subsection{Kurz gesagt}

Mangelnder Klimaschutz kann das Klimasystem in Zustände bringen, in denen Kipppunkte erreicht und Anpassungskapazitäten empfindlich überschritten werden. Während in Deutschland und Europa die Klimarisiken in einer Zwei-Grad-Welt durch optimale Anpassung bis 2100 theoretisch noch auf ein mittleres bis geringes Niveau begrenzt werden können, liegen die Risiken für andere Weltregionen wie beispielsweise die Nicht-OECDLänder in südlichen Regionen auch bei optimaler Anpassung hoch bis sehr hoch und damit in der Nähe harter Grenzen der Anpassung. Zur Einhaltung des Zwei-Grad-Zieles wird allerdings ein Ausmaß an Klimaschutz notwendig sein, das über inkrementelle Strukturanpassungen weit hinausgehen muss: Es bedarf einer "großen Transformation“ nationaler und globaler Wirtschaftsweisen, Rahmenbedingungen und Entwicklungspfade, sodass für die Nicht-OECD-Länder die Dekarbonisierung in den Mittelpunkt der Entwicklung rücken kann. Unter anderem sind eine konsequente Dekarbonisierung der Energiesysteme, der Landnutzung, des Wohnens und der Mobilität erforderlich. Das Abkommen der COP21 im Dezember 2015 in Paris ist ein wichtiger Schritt in diese Richtung.

Doch schon auf nationaler Ebene stehen gesellschaftliche Akteure und politische Entscheidungsträger vor komplexen Analyse- und Steuerungsproblemen. Um Klimarisiken zu begegnen und Chancen der Klimaanpassung auszuschöpfen, müssen die verschiedenen Dimensionen wirtschaftlicher, sozialer, politischer und ökologischer Systeme, räumliche und zeitliche Skalenebenen berücksichtigt und Praxisakteure einbezogen werden. Erweiterte Kosten-Nutzen-Analysen, die diese Vielzahl von Systemdimensionen und Skalenebenen einbeziehen oder auch mit Multikriterienanalysen gekoppelt werden können, sind geeignet, politische Akteure bei der Entscheidungsfindung zu unterstützen.

Eine Abkopplung der Wohlstandentwicklung von Emissionen und damit eine Vermeidung von rebound-Effekten kann jedoch nur gelingen, wenn sich zugleich veränderte Lebensstile und Konsummuster, neue Wohlfahrtskonzepte sowie Normen und Wertesysteme durchsetzen, die den Erhalt der globalen Gemeinschaftsgüter als unverzichtbar begreifen. Nur so kann eine Transformation in Richtung einer klimaresilienten Gesellschaft angestoßen, umgesetzt und verstetigt werden.

\section{Literatur}

Adger WN, Dessai S, Goulden M, Hulme M, Lorenzoni I, Nelson DR, Naess LO, Wolf J, Wreford A (2009) Are there social limits to adaptation to climate change? Clim Change 93:335-354

Allen MR, Frame DJ, Huntingford C, Jones CD, Lowe JA, Meinshausen M, Meinshausen N (2009) Warming caused by cumulative carbon emissions towards the trillionth tonne. Nature 458:1163-1166

Biermann F (2007) „Earth system governance" as a crosscutting theme of global change research. Glob Environ Chang 17:326-337

Biermann F (2008) Earth system governance. A research agenda. In: Young OR, King LA, Schroeder H (Hrsg) Institutions and environmental change. Principal findings, applications, and research frontiers. MIT Press, Cambridge, S 277-301 
Bosello F, Carraro C, De Cian E (2010) Climate policy and the optimal balance between mitigation, adaptation and unavoided damage. Clim Chang Econ 1:71-92

Brand U, Brunnengräber A, Andresen S, Driessen P, Haberl H, Hausknost D, Helgenberger S, Hollaender K, Læssøe J, Oberthür S, Omann I, Schneidewind U (2013) Debating transformation in multiple crises. ISSC/UNESCO (2013) World social science report 2013: changing global environments. OECD Publishing and UNESCO Publishing, Paris

Bruin KC de, Dellink RB, Tol RS (2009) AD-DICE: an implementation of adaptation in the DICE model. Clim Chang 95:63-81

Chum H, Faaij A, Moreira J, Berndes G, Dhamija P, Dong H, Gabrielle B, Eng AG, Lucht W, Mapako M, Cerutti OM, Mclntyre T, Minowa T, Pingoud K (2011) Bioenergy. In: Edenhofer O, Pichs-Madruga R, Sokona $Y$, Seyboth K, Matschoss P, Kadner S, Zwickel T, Eickemeier P, Hansen G, Schlömer S, Stechow C von (Hrsg) Climate Change 2014. Cambridge University Press, Cambridge

Cramer W, Yohe GW, Auffhammer M, Huggel C, Molau U, da Silva Dias MAF, Solow A, Stone DA, Tibig $L$ (2014) Detection and attribution of observed impacts. Climate change 2014: impacts, adaptation, and vulnerability. Part A: Global and sectoral aspects. Contribution of Working Group II to the Fifth Assessment Report of the Intergovernmental Panel on Climate Change. Cambridge University Press, Cambridge, S 979-1037

Crutzen P (2000) The Anthropocene. Glob Chang Newsletter 41:17-18

Defra, Scottish Government, Welsh Government, Department of the Environment Northern Ireland (2012) The UK climate change risk assessment report 2012. Government report, London

Depledge J (2005) The organization of global negotiations: constructing the climate change regime. Earthscan, London

Dessai S, Hulme M (2007) Assessing the robustness of adaptation decisions to climate change uncertainties: a case study on water resources management in the East of England. Glob Environ Chang 17:59-72

Duarte CM, Lenton TM, Wadhams P, Wassmann P (2012) Abrupt climate change in the Arctic. Nat Clim Chang 2:60-62

Edenhofer O, Stern N (2009) Towards a global green recovery - recommendations for immediate G20 action. A study initiated by the Federal Foreign Office and carried out by the Potsdam Institute for Climate Impact Research and the London School of Economics. Potsdam-Institut für Klimafolgenforschung, Potsdam

Edenhofer O, Carraro C, Hourcade JC, Neuhoff K, Luderer G, Flachsland C, Jakob M, Popp A, Steckel J, Strohschein J, Bauer N, Brunner S, Leimbach M, LotzeCampen H, Bosetti V, de Cian E, Tavoni M, Sassi O, Waisman H, CrassousDoerfler R, Monjon S, Droege S, van Essen H, del Río P, Tuerk A (2009) The economics of decarbonisation. Report of the RECIPE Project. PIK, Potsdam

EWI - Energiewirtschaftliches Institut an der Universität Köln, Prognos AG und GWS - Gesellschaft für Wirtschaftliche 393 Strukturforschung (2010) Energieszenarien für ein Energiekonzept der Bundesregierung. Projekt Nr. 12/10. EWI, Prognos, GWS, Köln

Fankhauser S (2010) The costs of adaptation. Wiley interdisciplinary reviews: climate change 1:23-30

Fisher-Vanden K, Wing IS, Lanzi E, Popp D (2013) Modeling climate change feedbacks and adaptation responses: recent approaches and shortcomings. Climatic Change (2013) 117 (3):481-495

Folke C, Carpenter SR, Walker B, Scheffer M, Chapin T, Rockström J (2010) Resilience thinking: integrating resilience, adaptability and transformability. Ecol Society 15(4):20

Frieler K, Meinshausen M, Golly A, Mengel M, Lebek K, Donner SD, Hoegh-Guldberg $\mathrm{O}$ (2012) Limiting global warming to $2{ }^{\circ} \mathrm{C}$ is unlikely to save most coral reefs. Nat Clim Chang 3:165-170

GDV Gesamtverband der Deutschen Versicherungswirtschaft e.V. (2013) Naturgefahrenreport 2013. http://www.gdv.de/wp-content/uploads/2014/08/ GDV_Naturgefahrenreport_2013n.pdf. Zugegriffen: 30. Mai 2016

\section{Gesang B (2011) Klimaethik. Suhrkamp, Frankfurt}

Giddens A (2009) The politics of climate change. Oxford University Press, Oxford Global Commission on the Economy and Climate (2014) The new climate economy. www.new climateeconomy.report

Grin J, Rotmans J, Schot J (2010) Transitions to sustainable development. New directions in the study of long term transformative change. Routledge, London
Guo J, Hepburn CJ, Tol RS, Anthoff D (2006) Discounting and the social cost of carbon: a closer look at uncertainty. Environ Sci Policy 9:205-216

Hallegatte S (2009) Strategies to adapt to an uncertain climate change. Global Environ Chang 19:240-247

Hallegatte S, Shah A, Lempert R, Brown C, Gill S (2012) Investment decision making under deep uncertainty. Background paper prepared for this report. World Bank, Washington DC

Hardin G (1968) The tragedy of the commons. Science 162:1243-1248

Hinkel J, Nicholls RJ, Vafeidis A, Tol RSJ, Avagianou T (2010) Assessing risk of and adaptation to sea-level rise in the European Union: an application of DIVA. Mitigation and adaptation strategies for global change 15(7):1-17

Hirschfeld J, von Möllendorff C (2015) Klimaökonomie braucht erweiterte Bewertungsmaßstäbe. Ökologisches Wirtschaften 30(1):23-25

Hirschfeld J, Pissarskoi E, Schulze S, Stöver J (2015) Kosten des Klimawandels und der Anpassung an den Klimawandel aus vier Perspektiven - Impulse der deutschen Klimaökonomie zu Fragen der Kosten und Anpassung. Hintergrundpapier zum 1. Forum Klimaökonomie des BMBF-Förderschwerpunktes "Ökonomie des Klimawandels", Berlin.

Hoegh-Guldberg O (2011) Coral reef ecosystems and anthropogenic climate change. Reg Environ Chang 11:215-227

Hübler M, Klepper G (2007) Kosten des Klimawandels. Die Wirkung steigender Temperaturen auf Gesundheit und Leistungsfähigkeit. Aktualisierte Fassung 07/2007. Arbeitspapier im Auftrag des WWF Deutschland, Frankfurt am Main

IEA - International Energy Agency (2010) Energy Balances of IEA Countries. IEA, Paris

IEA - International Energy Agency (2013) World Energy Outlook 2013. Organization for Economic Co-operation and Development (OECD), London

lerland EC van, Bruin K de, Watkiss P (2013) Multi-criteria analysis: decision support methods for adaptation. MEDIATION Project. Briefing Note 6:S 1-9

IPCC (2012) Meeting report of the intergovernmental panel on climate change. Expert meeting on Geoengineering. IPCC, Genf., S 99

IPCC (2013) Summary for Policymakers. In: Stocker TF, Qin D, Plattner G, Tignor M, Allen SK, Boschung J, Nauels A, Xia Y, Bex V, Midgley PM (Hrsg) Climate Change 2013: The physical science basis. Contribution of Working Group I to the Fifth Assessment Report of the Intergovernmental Panel on Climate Change. Cambridge University Press, Cambridge, S 33

IPCC (2014a) Climate Change 2014: Mitigation of climate change. Contribution of Working Group III to the Fifth Assessment. Report of the Intergovernmental Panel on Climate Change. Edenhofer O, Pichs-Madruga R, Sokona Y, Farahani E, Kadner S, Seyboth K, Adler A, Baum I, Brunner S, Eickemeier P, Kriemann B, Savolainen J, Schlömer S, von Stechow C, Zwickel T, Minx JC (Hrsg) Cambridge University Press, Cambridge, United Kingdom and New York, NY, USA

IPCC (2014b) Climate Change 2014. Synthesis Report. Contribution of Working Groups I, II and III to the Fifth Assessment Report of the Intergovernmental Panel on Climate Change. IPCC, Genf. (Core Writing Team, R.K. Pachauri and L.A. Meyer (Hrsg))

IPCC (2014c) Summary for Policymakers. Field CB et al (Hrsg) Climate change 2014: impacts, adaptation and vulnerability. Contribution of Working Group II to the Fifth Assessment Report of the Intergovernmental Panel on Climate Change. Cambridge University Press, Cambridge

Jackson T (2009) Prosperity without growth. Routledge, London

Jahn T (2008) Transdisziplinarität in der Forschungspraxis. In: Bergmann M, Schramm E (Hrsg) Transdisziplinäre Forschung. Integrative Forschungsprozesse verstehen und bewerten. Campus Verlag, Frankfurt, S 21-37

Jakob M, Steckel JC (2014) How climate change mitigation could harm development in poor countries. WIREs Clim Chang 5:161-168

JPI (Joint Programming Initiative) Climate (Hrsg) (2011) Strategic Research Agenda. Helsinki.

Kaplinsky R, Messner D (2008) The impacts of Asian drivers on the developing world. World Development 36(2):197-209

Keohane RO, Victor DG (2010) The regime complex for climate change. The Harvard Project on International Climate Agreements. Harvard University, Cambridge

Kharas $\mathrm{H}$ (2010) The emerging middle class in developing countries. OECD Development Centre Working Paper, Bd. 285. 
Klein RJT, Huq S, Denton F, Downing TE, Richels RG, Robinson JG, Toth FL (2007) Inter-relationships between adaptation and mitigation. In: Parry ML, Canziani OF, Palutikof JP, van der Linden PJ, Hanson CE (Hrsg) Climate Change 2007: Impacts, adaptation and vulnerability. Contribution of Working Group II to the Fourth Assessment Report of the Intergovernmental Panel on Climate Change. Cambridge University Press, Cambridge, S 745-777

Kovats S, Valentini R, Bouwer LM, Georgopoulou E, Jacob D, Martin E, Rounsevell M, Soussana JF et al (2014) Europe. In: Field CB (Hrsg) Climate change 2014: impacts, adaptation and vulnerability. Cambridge University Press, Cambridge

Kriegler E, O'Neill BC, Hallegatte S, Kram T, Lempert RJ, Moss RH, Wilbanks T (2012) The need for and use of socio-economic scenarios for climate change analysis: a new approach based on shared socio-economic pathways. Glob Environ Chang 22:807-822

Leggewie C, Messner D (2012) The low-carbon transformation: a social science perspective. J Renew Sus Energy 4:

Lehr U, Nieters A (2015) Makroökonomische Bewertung von Extremwetterereignissen in Deutschland. Ökologisches Wirtschaften 30.1:18-20

Lenton TM (2012) Arctic climate tipping points. AMBIO: J Human Environ 41:10-22

Lenton TM, Held H, Kriegler E, Hall JW, Lucht W, Rahmstorf S, Schellnhuber HJ (2008) Tipping elements in the Earth's climate system. PNAS 105:17861793

Levermann A, Bamber JL, Drijfhout S, Ganopolski A, Haeberli W, Harris NR, Huss M, Krüger K, Lenton TM, Lindsay RW (2012) Potential climatic transitions with profound impact on Europe. Clim Chang 110:845-878

Luderer G, Pietzcker RC, Bertram C, Kriegler E, Meinshausen M, Edenhofer O (2013) Economic mitigation challenges: how further delay closes the door for achieving climate targets. Environ Res Letters 8:034033

Martens P, Rotmans J (2002) Transitions in a globalizing world. Swets \& Zeitlinger Publishers, Tokio

Meinshausen M, Meinshausen N, Hare W, Raper SCB, Frieler K, Knutti R, Frame DJ, Allen MR (2009) Greenhouse-gas emission targets for limiting global warming to $2^{\circ} \mathrm{C}$. Nature 458:1158-1161

Messner D (2015) A social contract for low carbon and sustainable development - reflections on non-linear dynamics of social realignments and technological innovations in transformation processes. Technol Forecasting Social Chang 98(9):260-270

Messner D, Weinlich S (2016) The evolution of human cooperation: lessons learned for the future of global governance. In: Messner D, Weinlich S (Hrsg) Global cooperation and the human factor in international relations. Routledge, New York, S 3-46

Moser SC (2012) Adaptation, mitigation, and their disharmonious discontents: an essay. Clim Chang 111:165-175

Moser SC, Jeffress Williams S, Boesch DF (2012) Wicked challenges at Land's End: managing coastal vulnerability under climate change. Annu Rev Environ Resources 37:51-78

Nakicenovic N, Alcamo J, Davis G, Vries B de, Fenhann J, Gaffin S, Gregory K, Grübler A, Jung TY, Kram T, Lebre La Rovere E, Michaelis L, Mori S, Morita T, Pepper W, Pitcher H, Price L, Riahi K, Roehrl A, Rogner H-H, Sankovski A, Schlesinger M, Priyadarshi S, Smith S, Swart R, Rooijen S van, Victor N, Dadi Z (2000) Special report on emissions scenarios. Working Group III. Cambridge University Press, Cambridge

Newton-Smith WH (1980) The structure of time. Routledge, London

Nordhaus WD (2013) The climate casino. Yale University Press, New Haven

Oberthuer S, Gehring T (2005) Reforming international environmental governance: an institutional perspective on proposals for a world environment organization. In: Biermann F, Bauer S (Hrsg) A world environment organization: solution or threat for effective international environmental governance?. Ashgate, Aldershot, S 205-235

OECD - Organization for Economic Co-operation and Development (2010) Perspectives on global development 2010: shifting wealth. OECD, Paris

Olson M (1965) The logic of collective action: public goods and the theory of groups. Harvard University Press, Cambridge

Osterhammel J (2009) Die Verwandlung der Welt. Eine Geschichte des 19. Jahrhunderts. Beck, München
Ostrom E (2009) A polycentric approach for coping with climate change. Background paper to the 2010 World Development Report. Policy Research Paper 5095. World Bank, Washington

Ostrom E (2010) Polycentric systems for coping with collective action and global environmental change. Glob Environ Chang 20:550-557

Ott HE, Sterk W, Watanabe R (2008) The Bali roadmap: new horizons for global climate policy. Clim Policy 8:91-95

Pan J (2009) Carbon budget proposal. Research Center for Sustainable Development. Chinese Academy of Social Sciences, Peking

Parry M (2009) Closing the loop between mitigation, impacts and adaptation. Clim Chang 96:23-27

Parry M, Lowe J, Hanson C (2009) Overshoot, adapt and recover. Nature 458:1102-1103

Patt AG, van Vuuren DP, Berkhout F, Aaheim A, Hof AF, Isaac M, Mechler R (2010) Adaptation in integrated assessment modeling: where do we stand? Clim Chang 99:383-402

Pörtner H-O, Karl D, Boyd PW, Cheung W, Lluch-Cota SE, Nojiri Y, Schmidt DN, Zavialov P (2014) Ocean systems. In: Field CB, Barros VR, Dokken DJ, Mach KJ, Mastrandrea MD, Bilir TE, Chatterjee M, Ebi KL, Estrada YO, Genova RC, Girma B, Kissel ES, Levy AN, MacCracken S, Mastrandrea PR, White LL (Hrsg) Climate Change 2014: Impacts, Adaptation, and Vulnerability. Part A: Global and sectoral aspects. Contribution of Working Group II to the Fifth Assessment Report of the Intergovernmental Panel on Climate Change. Cambridge University Press, Cambridge, United Kingdom and New York, NY, USA, S 411-484

Post E, Forchhammer MC, Bret-Harte MS, Callaghan TV, Christensen TR, Elberling B, Fox AD, Gilg O, Hik DS, Høye TT, Ims RA, Jeppesen E, Klein DR, Madsen J, McGuire AD, Rysgaard S, Schindler DE, Stirling I, Tamstorf MP, Tyler NJC, van der Wal R, Welker J, Wookey PA, Schmidt NM, Aastrup P (2009) Ecological dynamics across the Arctic associated with recent climate change. Science 325:1355-1358

Preston BL, Dow K, Berkhout F (2013) The climate adaptation frontier. Sustainability 5:1011-1035

Robine JM, Cheung SL, Le Roy S, Van Oyen H, Griffiths C, Michel JP, Herrmann FR (2008) Death toll exceeded 70,000 in Europe during the summer of 2003. C R Biol 331(2):171-178

Rotmans J, Kemp R, van Asselt M (2001) More evolution than revolution: transition management in public policy. J Futures Stud. Strat Thinking Pol 3:15-31

Schellnhuber H-J (1999) Earth system analysis and the second Copernican Revolution. Eingeladener Beitrag für das „Supplement to Nature", 402 (6761), C19-C23

Schmitz H, Johnson O, Altenburg T (2013) Rent management. The heart of green industrial policy. IDS Working Paper, Bd. 418. IDS, Brighton

Schröder A, Hirschfeld J (2015) Tourismus im Klimawandel - Dynamische Input-Output-Modellierung der deutschen Ostseeregion. Ökologisches Wirtschaften 30.1:26-27

Sieferle RP, Krausmann F, Schandl H, Winiwarter V (2006) Das Ende der Fläche. Zum gesellschaftlichen Stoffwechsel der Industrialisierung. Boehlau, Köln

Sinn HW (2008) Public policies against global warming: a supply side approach. International Tax and Public Finance 15:360-394

Skidelsky R, Skidelsky E (2012) How much is enough? Money and the good life. Allen Lane, London (dt. Übers. (2013). Wie viel ist genug? Kunstmann, München)

Smith MS, Horrocks L, Harvey A, Hamilton C (2011) Rethinking adaptation for a 4 C world. Stern N (2013) The structure of economic modeling of the potential impacts of climate change: grafting gross underestimation of risk onto already narrow science models. J Econ Lit 51:838-859

Spence M (2011) The next convergence: the future of economic growth in a multispeed world. Farrar, Straus and Giroux, New York

Stern N (2013) The structure of economic modeling of the potential impacts of climate change: grafting gross underestimation of risk onto already narrow science models. J Econ Lit 51:838-859

Tröltzsch J, Görlach B, Lückge H, Peter M, Sartorius C (2011) Ökonomische Aspekte der Anpassung an den Klimawandel. Literaturauswertung zu Kosten und Nutzen von Anpassungsmaßnahmen an den Klimawandel. Clim Chang, Bd. 19. Umweltbundesamt, Dessau-Roßlau 
Kapitel 31 - Die klimaresiliente Gesellschaft - Transformation und Systemänderungen

UN Habitat (2011) Global report on human settlements - cities and climate change. Routledge, New York

UNDP - (United Nations Development Programme (2013) Human development report - the rise of the South. UNDP, New York

Urban F, Nordensvard J (2013) Low carbon development: key issues. Routledge, London

Vetter A, Schauser I (2013) Adaptation to climate change: prioritizing measures in the German adaptation strategy. GAIA 22(4):248-254

Van Vuuren DP, Isaac M, Kundzewicz ZW, Arnell N, Barker T, Criqui P, Berkhout F, Hilderink H, Hinkel J, Hof A (2011) The use of scenarios as the basis for combined assessment of climate change mitigation and adaptation. Glob Environ Chang 21:575-591

Walker BC, Holling S, Carpenter SR, Kinzig A (2004) Resilience, adaptability and transformability in social-ecological systems. Ecol Soc 9(2):5

Warren R (2011) The role of interactions in a world implementing adaptation and mitigation solutions to climate change. Phil Trans R Soc A 369:217-241

Wassmann P, Lenton TM (2012) Arctic tipping points in an Earth system perspective. Ambio 41:1-9

Watkiss P (2009) Potential costs and benefits of adaptation options: a review of existing literature. UNFCCC Technical paper 2009/2. Eigenverlag, Bonn

WBGU - Wissenschaftlicher Beirat der Bundesregierung Globale Umweltveränderungen (2006) Die Zukunft der Meere - zu warm, zu hoch, zu sauer. Sondergutachten 2006. WBGU, Berlin

WBGU - Wissenschaftlicher Beirat der Bundesregierung Globale Umweltveränderungen (2009) Kassensturz für den Weltklimavertrag - Der Budgetansatz. WBGU, Berlin

WBGU - Wissenschaftlicher Beirat der Bundesregierung Globale Umweltveränderungen (2011) Welt im Wandel - Gesellschaftsvertrag für eine Große Transformation. WBGU, Berlin

WBGU - Wissenschaftlicher Beirat der Bundesregierung Globale Umweltveränderungen (2014) Klimaschutz als Weltbürgerbewegungen. WBGU, Berlin

Weitzman ML (2009) On modeling and interpreting the economics of catastrophic climate change. Rev Econ Stat 91:1-19

Weitzman ML (2013) Tail-hedge discounting and the social cost of carbon. J Econ Lit 51:873-882

Wilby RL, Dessai S (2010) Robust adaptation to climate change. Weather 65:180-185

Williams M, Zalasiewicz J, Haywood A, Ellis M (2011) Special theme issue: The Anthropocene - e new epoch of geological time. Phil Trans R Soc 369:842867

World Bank (2010) Economics of adaptation to climate change - synthesis report. World Bank, Washington DC, S 136

World Bank (2012) Inclusive green growth - the pathway to sustainable development. The World Bank, Washington DC

Zimmerman D (2005) The A-theory of time, the B-theory of time, and taking tense seriously. Dialectica 59:401-457

Open Access Dieses Kapitel wird unter der Creative Commons Namensnennung 4.0 International Lizenz (http://creativecommons.org/ licenses/by/4.0/deed.de) veröffentlicht, welche die Nutzung, Vervielfältigung, Bearbeitung, Verbreitung und Wiedergabe in jeglichem Medium und Format erlaubt, sofern Sie den/die ursprünglichen Autor(en) und die Quelle ordnungsgemäß nennen, einen Link zur Creative Commons Lizenz beifügen und angeben, ob Änderungen vorgenommen wurden.

Etwaige Abbildungen oder sonstiges Drittmaterial unterliegen ebenfalls der genannten Creative Commons Lizenz, sofern sich aus der Abbildungslegende oder der Quellreferenz nichts anderes ergibt. Sofern solches Drittmaterial nicht unter der genannten Creative Commons Lizenz steht, ist eine Vervielfältigung, Bearbeitung oder öffentliche Wiedergabe nur mit vorheriger Zustimmung des betreffenden Rechteinhabers oder auf der Grundlage einschlägiger gesetzlicher Erlaubnisvorschriften zulässig. 